\title{
Hourly Average Wind Speed Simulation and Forecast Based on ARMA Model in Jeju Island, Korea
}

\author{
Duy-Phuong N. Do*, Yeonchan Lee* and Jaeseok Choi ${ }^{\dagger}$
}

\begin{abstract}
This paper presents an application of time series analysis in hourly wind speed simulation and forecast in Jeju Island, Korea. Autoregressive - moving average (ARMA) model, which is well in description of random data characteristics, is used to analyze historical wind speed data (from year of 2010 to 2012). The ARMA model requires stationary variables of data is satisfied by power law transformation and standardization. In this study, the autocorrelation analysis, Bayesian information criterion and general least squares algorithm is implemented to identify and estimate parameters of wind speed model. The ARMA $(2,1)$ models, fitted to the wind speed data, simulate reference year and forecast hourly wind speed in Jeju Island.
\end{abstract}

Keywords: Autoregressive moving average processes, Autoregressive processes, Autocorrelation function, Time series model, Wind energy, Wind speed forecast, Wind speed simulation

\section{Introduction}

Nowadays, the penetration of wind power continues to increase in power system, especially in Korea. Wind is considered as an energy credit but it is also fluctuation. It needs to access economic and technological factors. The study of wind power forecast, comprehensive reliability and cost evaluation of the system is the answer. An essential step in the study is to model the wind speed of specific site. The wind speed model is used to reproduce accurately actual wind speed. The analysis of historical wind speed data is to determine the necessary parameters for the model.

Data of hourly average wind speed (HAWS) in a specific location is time series, which has the following basic characteristics. Firstly, it is numeric statistics whose characteristics are illustrated by specific parameters such as probability distribution, mean value, standard deviation. Secondly, the values of time series have a correlation that means the relation of the present time value to past time values. This correlation is reflected by auto-correlation functions (ACF) and partial auto-correlation functions (PACF). Ones of the favorite methods in modeling correlation characteristics of time series is Box-Jenkins methodology or Autoregressive moving average (ARMA) process. The ARMA models can use 3 years of historical HAWS data in Jeju Island and result in accurate shortterm forecast of HAWS [11]. There are three classes of ARMA processes. Autoregressive AR(p) process has characteristics that the present time data value is defined by $\mathrm{p}$ previous time values and white noise. Moving average

$\dagger$ Corresponding Author: Dept. of Electrical Engineering, ERI, RIGET, Gyeongsang National University, Korea. (jschoi@gnu.ac.kr)

* Dept. of Electrical Engineering, Gyeongsang National University,

Korea. (\{dndphuong, kkng1914\}@gnu.ac.kr)

Received: June 25, 2015; Accepted: July 28, 2016
$\mathrm{MA}(\mathrm{q})$ process has the present time data value which is defined by a weighted moving average of the noise pulse. And autoregressive moving average $\operatorname{ARMA}(p, q)$ process is mixed of the autoregressive and moving average characteristics [2].

Many previous studies had fitted ARMA process to wind speed data. These papers proposed the general approach for modeling wind speed [1] and case study of wind speed statistical models in specific site or multiple sites [3, $4,6,10-12]$. The wind speed data were almost transformed from Weibull distribution to normal distribution by Dubey method which the shape parameters of data is transformed to 3.6 and the power $\mathrm{m}$ for the transformation was specified by measure of distribution symmetry [1], Skewness method $[3,4,6,10]$ or by Box-Cox method which the power $\lambda$ for the transformation is specified by minimizing the Skewness of the data [8]. However, the transformed data was not checked the strongly stationary $[1,10]$ or instead of checking the strongly stationary of transformed data, normal distribution is only considered by comparing with corresponding normal probability density $[3,4,8]$ or testing non-stationary of transformed data by Duckey-fuller test [9]. These papers assumed that transformed data fitted to a $\mathrm{p}^{\text {th }}$ order autoregressive process $A R(p)$ [1] or the transformed data fitted to a $p^{\text {th }}$ order autoregressive process $\mathrm{AR}(\mathrm{p})$ by autocorrelation, partial autocorrelation analysis in identification process but there was no using Bayesian information criterion (BIC) to select an accurate class of ARMA(p,q) [3] or ACF, PACF and $\mathrm{BIC}$ were analyzed but the $\mathrm{AR}(\mathrm{p})$ was selected too soon after ACF and PACF analysis [8], instead of analyzing $\mathrm{ACF}, \mathrm{PACF}$ and $\mathrm{BIC}$ together or using only AIC $[9,10]$.

From above analyses, this paper presents ARMA model application to wind speed simulation and forecast in 


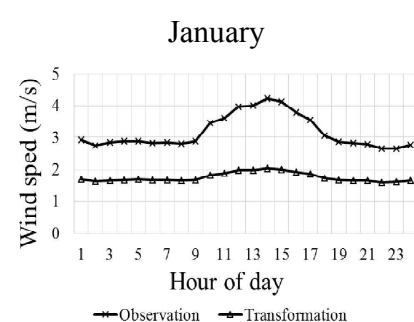

-Observaliou -Tramsumaliou

July

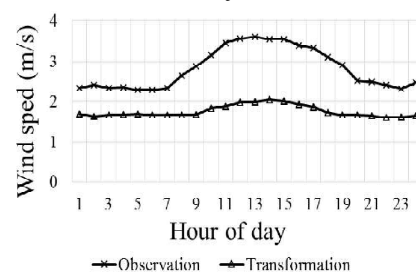

* Obscration $\rightarrow$ Transfomation

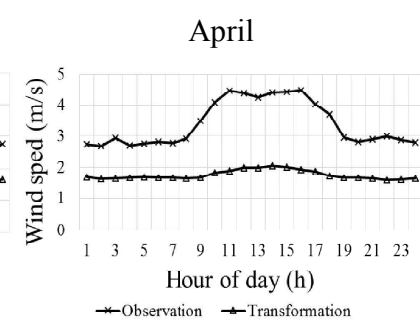

October

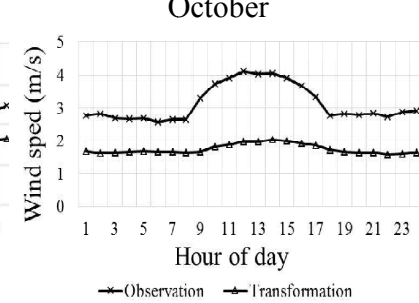

Fig. 1. The variation of observed and transformed hourly average wind speed in 24 hour periods of all day in a given month on Jeju Island, Korea (from 2010 to 2012 year)

specific site, Jeju Island, in Korea. The wind speed data of Jeju Island are not only Weibull distribution so that they are transformed to normal distribution by Box-Cox method, which the power $\lambda$ for the transformation is specified by likelihood function, the best suitable transformation. The new way is proposed for checking the strongly stationary of transformed data by combining $2^{\text {nd }}$ order stationary analysis and Q_Q plot "probability plot" for normal distribution check. The wind speed models are identified by analyzing $\mathrm{ACF}, \mathrm{PACF}$ and $\mathrm{BIC}$ together to select an accurate class of $\operatorname{ARMA}(p, q)$. In this paper, the 3 years' HAWS data from 2010 to 2012, which is in Seongsan meteorological station, is used.

\section{Stationary Data Transformation}

An important supposition in the build of the time series model is stationary because a stationary time series can easily be depicted by its mean, standard deviation or variance. ARMA model is only applied time series data which are normal distribution but there are no trend and seasonal components that mean stationary. There are some phases in simulating methodology. Firstly, the fit of ARMA models with suitable order is occurred to HAWS data which have been removed seasonal and daily nonstationary, and transformed to make their distribution approximately normality. Secondly, the parameters of models are estimated to optimize them. Finally, the fitted models are checked validly. The identification process for wind speeds is occurred by analyzing ACF and PACF that requires the stationary of data. It can be seen that HAWS observation $\mathrm{V}$ is not stationary (Fig. 1). This figure shows the daily variation of HAWS taken over 3 year (from 2010 to 2012) for all day of January, April, July and October which represent all four seasons of the year. The daily nonstationary can be removed from the standardization process to transform the data to normality distribution which is a mean of zero and variance of one $\mathrm{N}(0,1)[3]$.

\subsection{Normality data transformation}

The year of HAWS data is separated into monthly periods to remove the seasonal non-stationary [4]. Then, by fitting a separate model for each month, there are 12 models of HAWS in the reference year.

Because of non-normality distribution of HAWS data, there is no direct application of ARMA models to data. So, the transformation of HAWS using Box-Cox method is necessary [5]. Using the power parameter $\lambda$ and constant parameter $\mathrm{c}$ to transform HAWS $\mathrm{V}$ to $\mathrm{V}^{(\lambda)}$ by equation:

$$
V^{(\lambda)}=\left\{\begin{array}{c}
(V+c)^{\lambda} \text { for } \lambda \neq 0 \\
\ln (V+c) \text { for } \lambda=0
\end{array}\right.
$$

Assuming that there are some unknown $\lambda$, the transformed HAWS $\mathrm{V}_{i}^{(\lambda)}(\mathrm{i}=1, \ldots \mathrm{n})$ satisfy the normal distribution with constant variance $\widehat{\sigma}^{2}(\lambda)$. For fixed $\lambda$, maximum logarithm likelihood in relation to the observed wind speed achieved by [5]:

$$
\mathrm{L}_{\mathrm{max}}(\lambda)=-\frac{1}{2} n \ln \widehat{\sigma}^{2}(\lambda)+(\lambda-1) \sum \ln (\mathrm{V}+\mathrm{c})
$$

For various values of $\lambda$ from -2 to 2 , choosing $\lambda$ which results in the maximum $\log$ likelihood of $\mathrm{V}^{(\lambda)}$. To the wind speed data on Jeju Island, finding the maximum likelihood value is thus close to $\lambda=1.0$, identified data, in February, October and $\lambda=0.5$, root square data, for the others.

\subsection{The exclusion of daily HAWS fluctuation}

The daily fluctuation of transformed HAWS $\mathrm{V}^{(\lambda)}$ (Fig. 1) suggests that it is in need of exclusion by the standardization method which produces a standard normal distribution $\mathrm{N}(0,1)$. For a given month $\mathrm{m}$, having 24 hours $\mathrm{x}$ number of days of given month $\mathrm{x} 3$ years HAWS values. The mean of HAWS in the hour h (from 1 to 24) of day in given month $\mathrm{m}$ obtained from [3]:

$$
\mu(h, m)=\frac{1}{Y D} \sum_{y=1}^{Y} \sum_{d=1}^{D} V^{(\lambda)}(h, d, m, y)
$$

where $\mathrm{h}, \mathrm{d}, \mathrm{m}$, and $\mathrm{y}$ are the hour, the day, the month, the year, respectively. And $\mathrm{Y}$ is the number of years of observation and D is the number of days of a given month. Then, the variance of HAWS for the given month $m$ at hour h obtained from [3]: 


$$
\sigma^{2}(h, m)=\frac{1}{Y D} \sum_{y=1}^{Y} \sum_{d=1}^{D}\left[V^{(\lambda)}(h, d, m, y)-\mu(h, m)\right]^{2}
$$

From these $\mu(h, m)$ and $\sigma(h, m)$ series, the standardized and transformed HAWS is defined by [3]:

$$
\overline{\bar{V}}(\mathrm{~h}, \mathrm{~d}, \mathrm{~m}, \mathrm{y})=\frac{\mathrm{V}^{(\lambda)}(\mathrm{h}, \mathrm{d}, \mathrm{m}, \mathrm{y})-\mu(\mathrm{h}, \mathrm{m})}{\sigma(\mathrm{h}, \mathrm{m})}
$$

\subsection{Results of data transformation}

As above mentioned introduction section, the strongly stationary data is required condition of applying ARMA model [2]. The standardized and transformed HAWS in given month $\overline{\bar{V}}(\mathrm{~h}, \mathrm{~d}, \mathrm{~m}, \mathrm{y})$ are considered really stationary by 2 steps.

Firstly, checking $2^{\text {nd }}$ order stationary $\overline{\bar{V}}$ by the following expression:

$$
\rho_{\mathrm{n}}(\mathrm{h}, \mathrm{m})=\frac{1}{\mathrm{DY}-1} \sum_{\mathrm{y}=1}^{\mathrm{Y}} \sum_{\mathrm{d}=1}^{\mathrm{D}} \overline{\bar{V}}(\mathrm{~h}, \mathrm{~d}, \mathrm{~m}, \mathrm{y}) \cdot \overline{\bar{V}}(\mathrm{~h}+2, \mathrm{~d}, \mathrm{~m}, \mathrm{y})
$$

It reflects the approximation of the correlation function of $\overline{\bar{V}}$ between hour $\mathrm{h}$ and $\mathrm{h}+2$ of day in given month. Fig. 2 displays the correlation values of some months with second order. Most of them are within the $\rho_{\mathrm{n}}(\mathrm{m})$ $\pm \frac{1}{\sqrt{\mathrm{DY}}}$ values. Where $\rho_{\mathrm{n}}(\mathrm{m})$ and $\frac{1}{\sqrt{\mathrm{DY}}}$ are mean and standard deviation of $\rho_{\mathrm{n}}(\mathrm{h}, \mathrm{m})$ respectively. That means $\overline{\bar{V}}$ is second order stationary [2]. In Table 1, it also shows that the average of correlation values are within the limits. This step proves that the transformed data is stationary but weak. The transformed data need to consider in normal distribution.
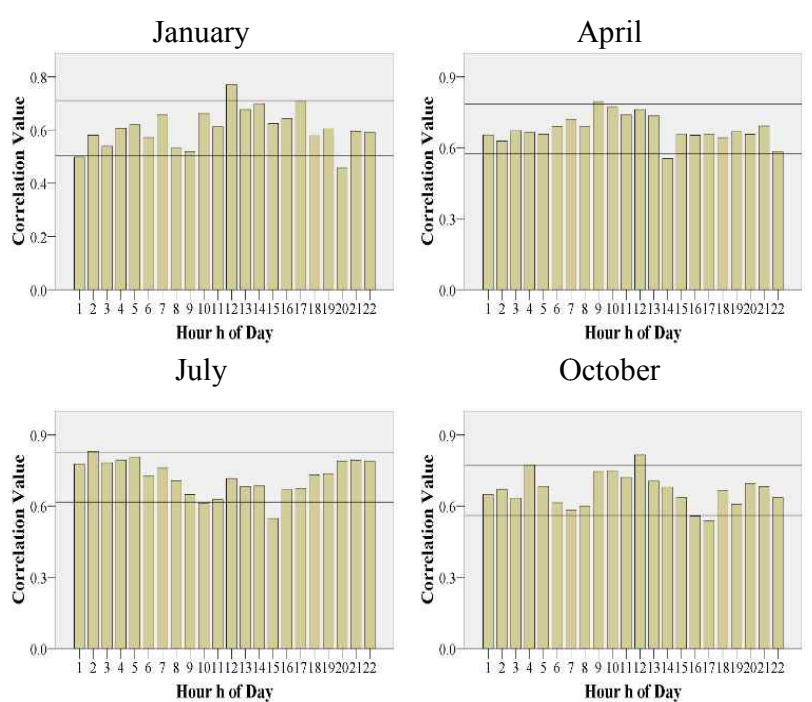

Fig. 2. The variation of autocorrelation function of second order for 24 hours for all day of given month
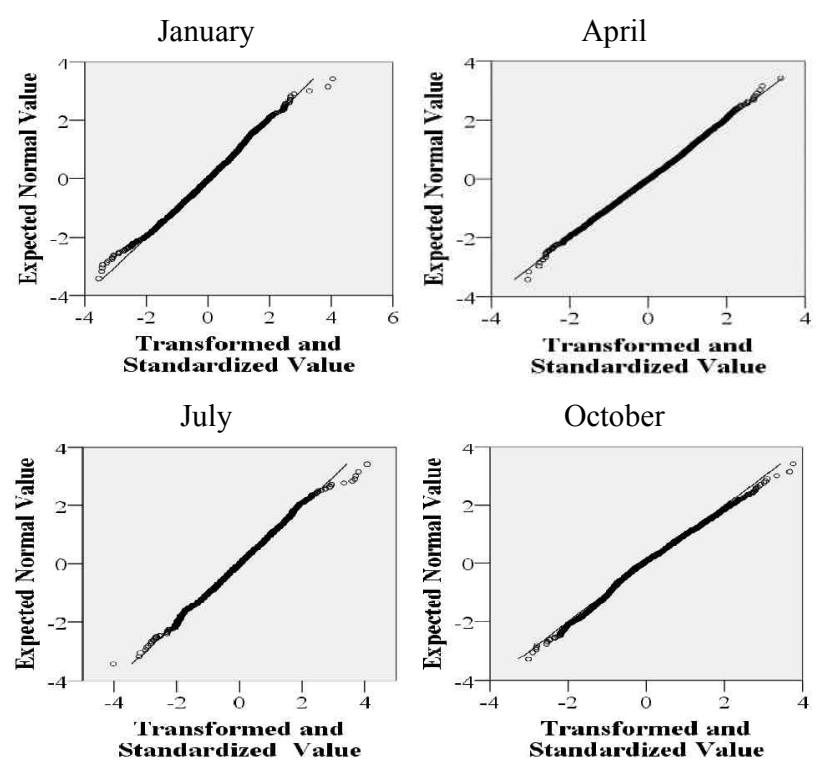

Fig. 3. The illustration of transformed HAWS data adapting to expected normal value

Table 1. Correlation values of transformed wind speed data

\begin{tabular}{c|c|c|c|c}
\hline Month & Average & $\begin{array}{c}\text { Standard } \\
\text { deviation }\end{array}$ & Lower limit & Upper limit \\
\hline January & 0.606 & 0.073 & 0.503 & 0.710 \\
\hline April & 0.679 & 0.058 & 0.574 & 0.784 \\
\hline July & 0.722 & 0.073 & 0.616 & 0.827 \\
\hline October & 0.665 & 0.070 & 0.560 & 0.771 \\
\hline
\end{tabular}

Secondly, $\overline{\bar{V}}$ is proved as normal distribution by Q-Q plot, called "probability plot". Fig.3 demonstrates the data is approximately adapted to expected normal value. From analyses, $\overline{\bar{V}}$ become strongly stationary [2] and the data are suitable for applying to ARMA model.

\section{Application of ARMA Model to Wind Speed Data in Jeju Island}

\subsection{ARMA model presentation}

The ARMA model is mix of autoregressive and moving average process. Where autoregressive process models the time series by taking into account the past values of the data and moving average process defines contingent data. By applying ARMA process, the standardized and transformed HAWS $\overline{\bar{V}}$ at present time $\mathrm{t}$ of time series of given month is expressed [2]:

$$
\overline{\bar{V}}_{t}=\sum_{i=1}^{p} \phi_{i} \cdot \overline{\bar{V}}_{t-i}+\alpha_{t}+\sum_{j=1}^{q} \theta_{j} \cdot \alpha_{t-j}
$$

where $\phi_{\mathrm{i}}, \theta_{\mathrm{j}}, \mathrm{p}$ and $\mathrm{q}$ are autoregressive, moving average parameters, and their orders respectively, $\alpha_{t}$ is white noise, random normal distribution with zero mean and $\sigma_{\alpha}^{2}$ 
variance.

\subsection{Wind speed model identification process}

Wind speed model is identified by autocorrelation and partial autocorrelation function analysis. In this stage, the order of models and initial estimation are defined. For a given month $\mathrm{m}$, the auto-covariance coefficients were estimated by Yule-Walker equations:

$$
\mathrm{c}_{\mathrm{k}}(\mathrm{m})=\frac{1}{\mathrm{HDY}-\mathrm{Yk}} \sum_{\mathrm{y}=1}^{\mathrm{Y}} \sum_{\mathrm{h}=1}^{\mathrm{HD}-\mathrm{k}} \overline{\bar{V}}(\mathrm{~h}, \mathrm{~m}, \mathrm{y}) \cdot \overline{\bar{V}}(\mathrm{~h}+\mathrm{k}, \mathrm{m}, \mathrm{y})
$$

where $\mathrm{H}=24$ hours of day. Then, estimates of the $\mathrm{k}^{\text {th }}$ lag autocorrelation coefficients is

$$
r_{k}(m)=\frac{c_{k}(m)}{c_{0}(m)}
$$

Applying this method to the months gives characteristic of ACF as in Fig. 4. After that the partial autocorrelation coefficients $\phi_{\mathrm{k}}$ is calculated from $\mathrm{r}_{\mathrm{k}}$ by equations [2]:

$$
\begin{aligned}
& \phi_{i}=\left\{\begin{array}{cl}
r_{1} & \text { for } i=1 \\
\frac{r_{1}-\sum_{j=1}^{i-1} \phi_{i-1, j} r_{i-j}}{1-\sum_{j=1}^{i-1} \phi_{i-1, j} r_{i-j}} & \text { for } i=2,3, \ldots, n
\end{array}\right. \\
& \phi_{\mathrm{j}}=\phi_{\mathrm{i}-1, \mathrm{j}}-\phi_{\mathrm{i}} \phi_{\mathrm{i}-1, \mathrm{i}-\mathrm{j}} \text { for } \mathrm{j}=1,2, \ldots, \mathrm{i}-1
\end{aligned}
$$

For each month, the ACF and PACF of $\overline{\bar{V}}$ show the plot of $r_{k}, \phi_{k}$ to $k$. The ACF exponentially lessen to zero (Fig. 4) while the PACF of months is zero after 2 or 3 lags (Fig. 5).

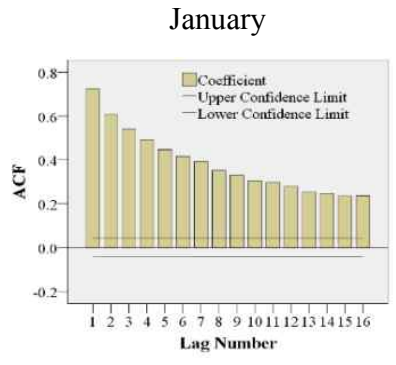

July

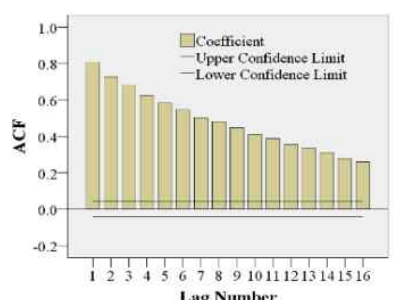

Lag Number

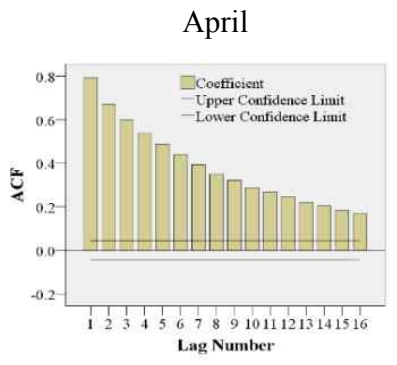

October

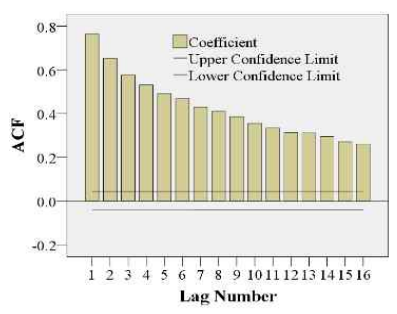

Lag Number
Fig. 4. Autocorrelation function of transformed \& standardized data
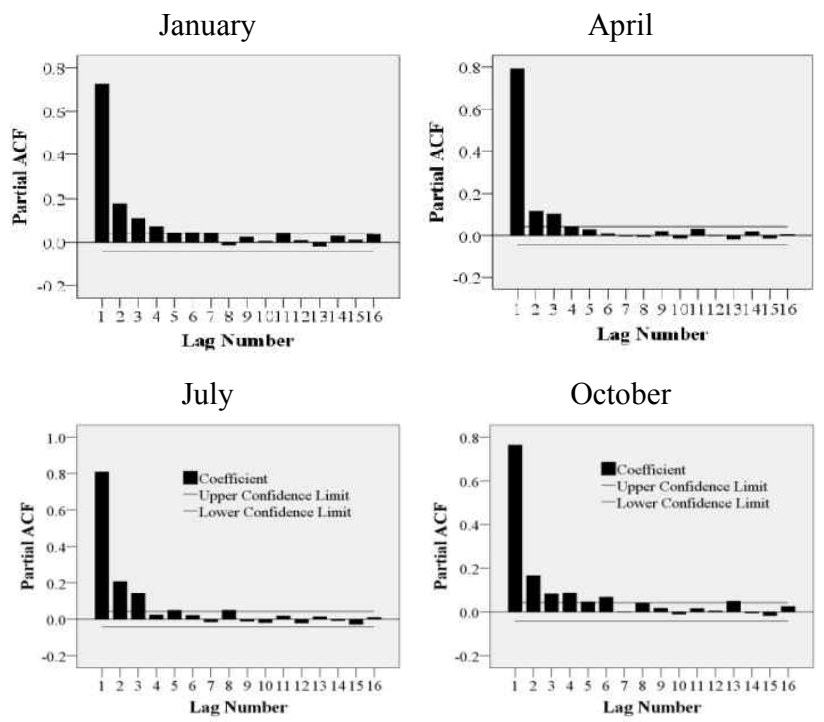

Fig. 5. Partial autocorrelation function of transformed\& standardized data

The shape of the ACF and PACF suggested ARMA(p,q) models with low order $\mathrm{p}$ and $\mathrm{q}(\mathrm{p}, \mathrm{q} \leq 3)$. Then, the Bayesian Information Criterion (BIC), based on likelihood function, is used to choose a model among a finite set of models. The values of order $\mathrm{p}$ and $\mathrm{q}$ are selected by minimizing the following expression:

$$
\mathrm{BIC}=(\mathrm{HDY}) \ln \left(\sigma_{\varepsilon}^{2}\right)+\mathrm{T} \ln (\mathrm{HDY})
$$

where $\mathrm{T}=\mathrm{p}+\mathrm{q}$ and $\sigma_{\varepsilon}^{2}$ is the variance of the residuals

The variance of the residuals $\sigma_{\varepsilon}^{2}$ is calculated by using following auto-covariance equations of $\operatorname{ARMA}(p, q)$ processes. These equations are also preliminarily to estimate the parameters $\phi_{\mathrm{i}}, \theta_{\mathrm{j}}$ of models [2].

$$
\begin{aligned}
\gamma_{\mathrm{k}}=\phi_{1} \gamma_{\mathrm{k}-1}+\cdots+\phi_{\mathrm{p}} \gamma_{\mathrm{k}-\mathrm{p}}+\gamma_{\mathrm{za}}(\mathrm{k}) \\
-\theta_{1} \gamma_{\mathrm{za}}(\mathrm{k}-1)-\cdots-\theta_{\mathrm{q}} \gamma_{\mathrm{za}}(\mathrm{k}-\mathrm{q})
\end{aligned}
$$

where $\gamma_{\mathrm{za}}$ is the cross covariance function between $\overline{\bar{V}}$ and residual $\varepsilon$ of guessed models, defined by taking expectation, and $\gamma_{\mathrm{k}}$ is the auto-covariance of ARMA processes, equals alternative calculation $c_{k}$ by the Yule-Walker equation in (8).

By (12) and (13), the mixed second order autoregressivefirst order moving average $\operatorname{ARMA}(2,1)$ model is fitted to each month. From (7) the $\operatorname{ARMA}(2,1)$ model is simplified to

$$
\overline{\bar{V}}_{t}=\phi_{1} \overline{\bar{V}}_{t-1}+\phi_{2} \overline{\bar{V}}_{t-2}-\theta_{1} \alpha_{t-1}+\alpha_{t}
$$

\subsection{Efficient estimates of parameters}

For obtaining more efficient estimates of the parameters $\phi_{1}, \phi_{2}, \theta_{1}$ in $\operatorname{ARMA}(2,1)$ models, the general least squares algorithm is applied. From the first guesses of the parameters $\phi_{1,0}, \phi_{2,0}, \theta_{1,0}$ in identification process, the initial calculation of white noise $\alpha_{t, 0}$ (15) and the 
Table 2. The parameter estimates of $\operatorname{ARMA}(2,1)$ models

\begin{tabular}{c|c|c|c|c|c}
\hline Months & $\phi_{1}$ & $\phi_{2}$ & $\theta_{1}$ & $\sigma_{\alpha}^{2}$ & Q-statistic \\
\hline Jan & 1.263 & -0.309 & 0.701 & 0.447 & 20.53 \\
\hline Feb & 1.200 & -0.263 & 0.597 & 0.405 & 28.35 \\
\hline Mar & 1.178 & -0.254 & 0.555 & 0.410 & 16.17 \\
\hline Apr & 1.304 & -0.360 & 0.626 & 0.359 & 18.34 \\
\hline May & 1.347 & -0.388 & 0.653 & 0.306 & 19.29 \\
\hline Jun & 1.236 & -0.281 & 0.620 & 0.335 & 30.23 \\
\hline Jul & 1.152 & -0.204 & 0.550 & 0.320 & 38.22 \\
\hline Aug & 1.086 & -0.132 & 0.437 & 0.221 & 33.25 \\
\hline Sep & 1.148 & -0.200 & 0.514 & 0.284 & 26.91 \\
\hline Oct & 1.340 & -0.376 & 0.729 & 0.393 & 24.74 \\
\hline Nov & 1.187 & -0.255 & 0.582 & 0.408 & 30.36 \\
\hline Dec & 1.166 & -0.229 & 0.599 & 0.418 & 36.34 \\
\hline
\end{tabular}

derivatives of $\alpha_{t}$ to parameter of models (16) and (17) obtained. Then, the starting values for $\alpha_{t}$ 's, $x_{t}$ 's, and $u_{t}$ 's series are set to zero and then calculated with the forward recursions, as follows [2]

$$
\begin{aligned}
\alpha_{t, 0} & =\overline{\bar{V}}_{t}-\phi_{1,0} \overline{\bar{V}}_{t-1}+\phi_{2,0} \overline{\bar{V}}_{t-2}+\theta_{1,0} \alpha_{t-1} \\
u_{t} & =\phi_{1,0} u_{t-1}+\phi_{2,0} u_{t-2}+\alpha_{t, 0} \\
x_{t} & =\theta_{1,0} x_{t-1}-\alpha_{t, 0}
\end{aligned}
$$

where $\alpha_{t, 0}$ is initial white noise, $\mathrm{u}_{\mathrm{t}}$ is the derivatives of $\alpha_{\mathrm{t}}$ to $\phi$, and $x_{t}$ is the derivatives of $\alpha_{t}$ to $\theta$.

Expanding expectation of $\alpha_{\mathrm{t}}$ in a Taylor series with first guesses of the parameters $\phi_{1,0}, \phi_{2,0}, \theta_{1,0}$, an approximate linear regression equation obtained:

$$
\begin{aligned}
\alpha_{t, 0}= & \left(\phi_{1}-\phi_{1,0}\right) \cdot u_{t-1}+\left(\phi_{2}-\phi_{2,0}\right) \cdot u_{t-2} \\
& +\left(\theta_{1}-\theta_{1,0}\right) \cdot x_{t-1}+\alpha_{t}
\end{aligned}
$$

By regressing $\alpha_{t, 0}$ on $u_{t-1}, u_{t-2}$, and $x_{t-1}$, the adjustment is added to the guesses of the parameters in every iteration step. From the new guesses of parameters, the new values of $\alpha_{t, 0}, u_{t-1}, u_{t-2}$, and $x_{t-1}$ are calculated by iteration process until convergence occurs. The results are as in Tables 2.

\subsection{Diagnostic checking of fitted model}

Final stage is to check the proposed models in table 2 by analyzing autocorrelation function of the residuals $r_{k}(\alpha)$. If the $\alpha_{t}$ 's series indicates the nature of models that means the $r_{k}(\alpha)$ will be uncorrelated and normal distribution, the fitted models are acceptable. Using "a portmanteau lack of fit test" to consider whether the first $\mathrm{K}=24$ autocorrelations of the residuals of $\operatorname{ARMA}(2,1)$ models are approximately distributed as Chi-square distribution (21 degrees of freedom). The Box-Pierce statistic (Q-statistic) is calculated by:

$$
\mathrm{Q}=\mathrm{HDY} \sum_{\mathrm{k}=1}^{\mathrm{K}} \mathrm{r}_{\mathrm{k}}^{2}(\alpha)
$$

Referring the results of Q-statistic in table 2 to Chisquare distribution with 21 degrees of freedom, the $5 \%$ and $1 \%$ points for $\chi^{2}$ distribution are 32.7 and 38.9 respectively. Therefore, all proposed model of months are acceptable.

\section{Simulation Results}

The ARMA(2,1) model for wind speeds is employed to generate a time series of simulated HAWS. Firstly, a sequence of independent random variables, denoted by $\alpha_{t}$, having normal distribution with zero mean and $\sigma_{\alpha}^{2}$ variance is generated for every month. Then, the time series of month $\overline{\bar{V}}_{t}$ is generated by (14). The simulated hourly wind speeds, denoted by $\tilde{V}_{1}, \tilde{V}_{2}, \ldots \tilde{V}_{t}$, are obtained as follows:

$$
\left.\tilde{V}_{t}=\left(\mu_{t}+\sigma_{t} * \overline{\bar{V}}_{t}\right)\right)^{2}
$$

where $\mu_{t}=\mu(\mathrm{h}, \mathrm{m})$ is the mean and $\sigma_{t}=\sigma(\mathrm{h}, \mathrm{m})$ is the standard deviation of hourly wind speed in the hour $h$ of given month.
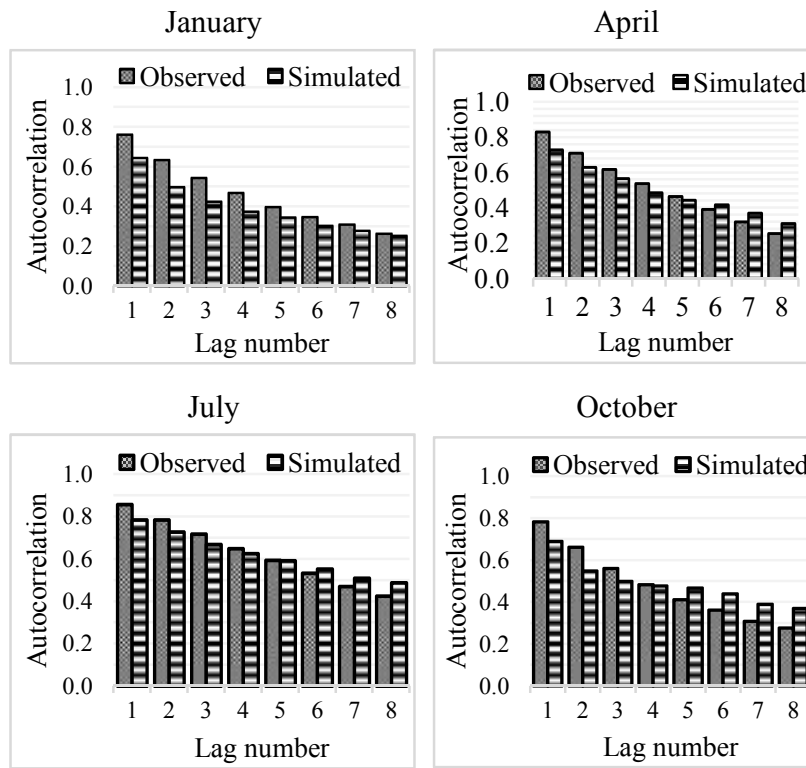

October

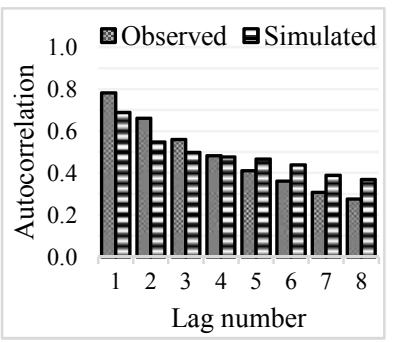

Fig. 6. Comparison of the observed and simulated autocorrelation functions
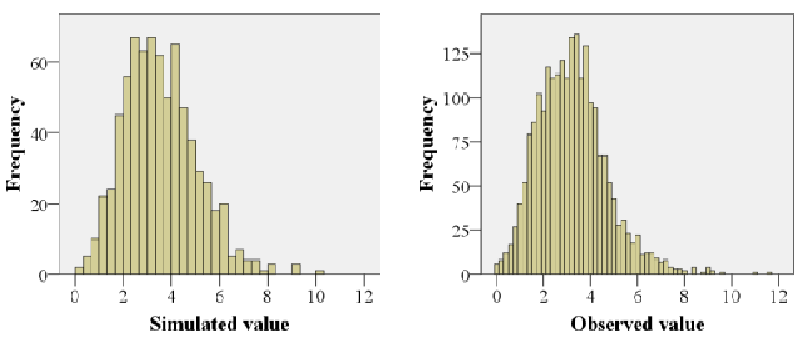

Fig. 7. Comparison of the observed and simulated frequency distributions of wind speed in January 
In consideration of checking the validity of the ARMA $(2,1)$ models, the comparison of wind speed simulation and HAWS observation is occurred by autocorrelation functions, frequency distribution and error percentages. Although autocorrelation coefficients of the simulation are slight underestimation but they are no statistical significance for all months (Fig. 6). It is proved that the fitted models generated approximate HAWS observations. Using the fitted ARMA $(2,1)$ model to produce a series of 24 hours $\times 31$ days $=744$ values of reference month of January. The histogram reveals main characteristics of wind speed simulations and observations in Fig. 7. The frequency distribution of 24 hours $\times 31$ days $\times 3$ years $=$ 2232 observed and 744 simulated values of January is also congruent that means the fit of the $\operatorname{ARMA}(2,1)$ model to actual wind speed data is very prospects. By the same way for each month, the fluctuation of average wind speed simulation in given months is presented in Fig. 8. The main characteristics of 3 years of HAWS observation is also reproduced by ARMA $(2,1)$.

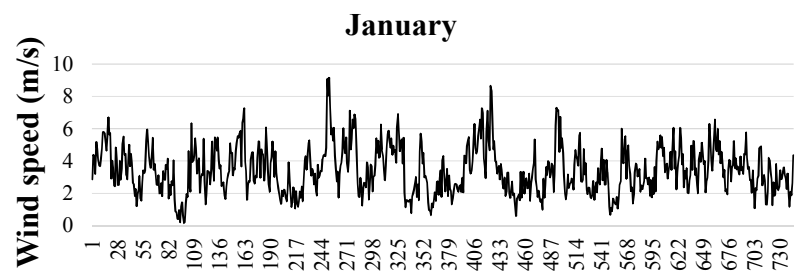

Hours in month (h)

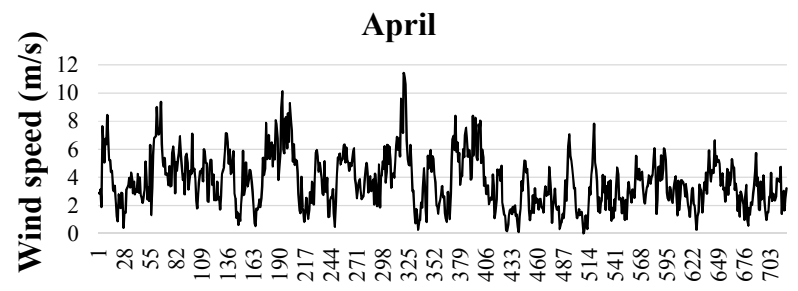

Hours in month (h)

July

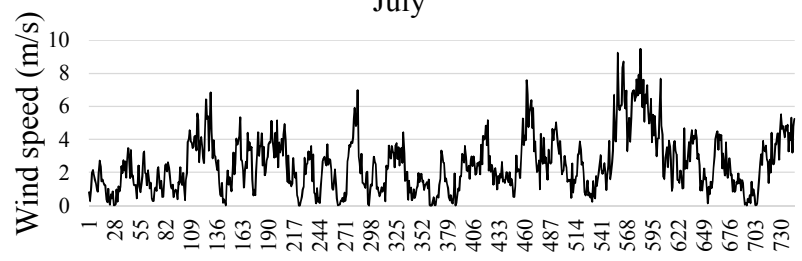

Hours in month (h)

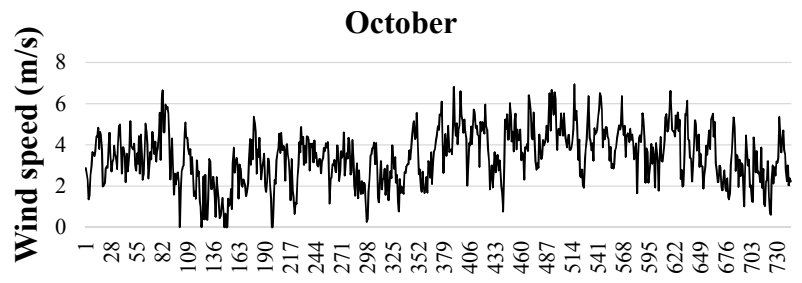

Hours in month (h)

Fig. 8. Hourly average wind speed simulation in given month
The comparison of hourly average wind speed simulations and observations of 24 hours periods of all day in given month and their standard deviations are as in Fig. 9. The simulated wind speed approximately revealed the daily variation characteristic and standard deviation of observed wind speed. It is also considered error of hourly average wind speed of 24 hours periods between the observation and simulation values in given month

$$
\text { error } \%=\frac{\mid \text { observation-simulation } \mid}{\text { observation }}
$$

The error is as in Fig. 9. The summary results for
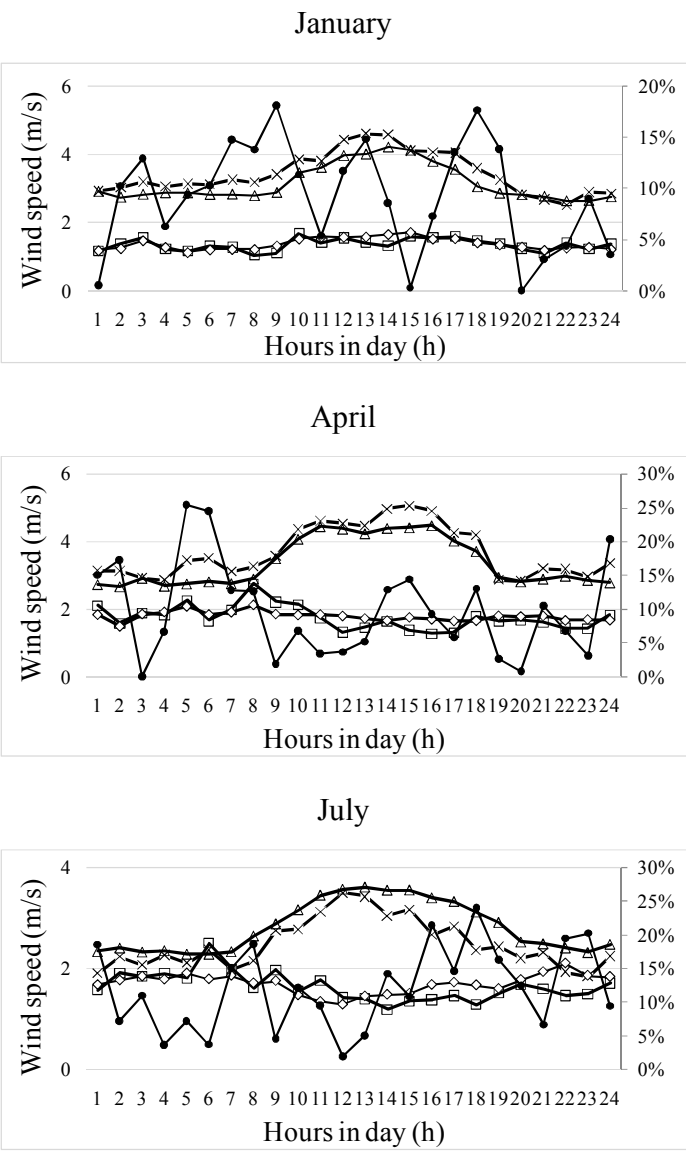

October

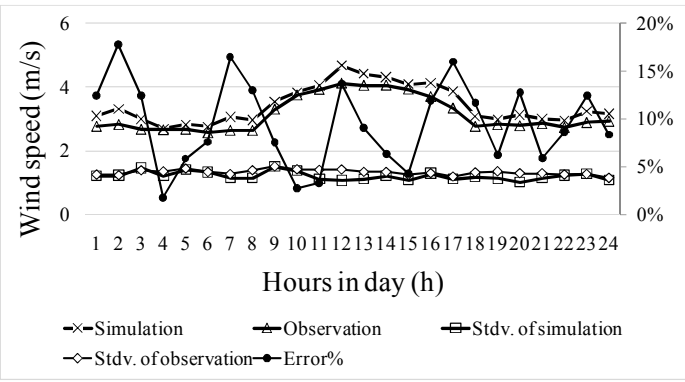

Fig. 9. The comparison of wind speed simulation and observation of $24 \mathrm{~h}$ of all day in given month 
Table 3. Statistics for Observed (Obs) and Simulated (Sim) HAWS in Jeju Island

\begin{tabular}{c|c|c|c|c|c|c}
\hline \multirow{2}{*}{ Month } & \multicolumn{3}{|c|}{ Mean $(\mathrm{m} / \mathrm{s})$} & \multicolumn{3}{c}{ Variance $(\mathrm{m} / \mathrm{s})^{2}$} \\
\cline { 2 - 7 } & Sim & Obs & Error\% & Sim & Obs & Error\% \\
\hline Jan & 3.44 & 3.16 & $8.7 \%$ & 2.19 & 2.17 & $1.1 \%$ \\
\hline Feb & 2.86 & 3.09 & $7.2 \%$ & 1.99 & 2.36 & $15.4 \%$ \\
\hline Mar & 3.74 & 3.65 & $2.6 \%$ & 2.72 & 3.06 & $10.8 \%$ \\
\hline Apr & 3.70 & 3.38 & $9.3 \%$ & 3.65 & 3.71 & $1.7 \%$ \\
\hline May & 3.10 & 2.99 & $3.4 \%$ & 3.11 & 3.11 & $0.2 \%$ \\
\hline Jun & 2.72 & 2.72 & $0.2 \%$ & 3.03 & 3.10 & $2.2 \%$ \\
\hline Jul & 2.49 & 2.82 & $11.8 \%$ & 2.92 & 3.15 & $7.2 \%$ \\
\hline Aug & 3.77 & 3.49 & $7.9 \%$ & 5.01 & 6.59 & $24.0 \%$ \\
\hline Sep & 3.34 & 3.18 & $5.2 \%$ & 3.14 & 3.27 & $3.8 \%$ \\
\hline Oct & 3.43 & 3.14 & $9.3 \%$ & 1.77 & 2.04 & $13.5 \%$ \\
\hline Nov & 2.65 & 3.02 & $12.2 \%$ & 1.92 & 2.35 & $18.3 \%$ \\
\hline Dec & 2.96 & 3.13 & $5.5 \%$ & 2.03 & 2.61 & $22.2 \%$ \\
\hline
\end{tabular}

synthetic sequences are presented in Table 3. From the above analysis and results of Table 2 shows that observation (obs) and simulation (sim) of hourly wind speed are satisfactory. The simulations of generating all months represent the approximate real statistical characteristics of 3 years of HAWS data in Jeju Island, Korea.

\section{Ahead of a Day Wind Speed Forecast}

The simulated ARMA $(2,1)$ model is also used to forecast hourly average wind speed in Jeju Island by the weighted sum of previous wind speed values [2]. The predicted wind speed is expressed as

$$
\widehat{V}_{t}(l)=\pi_{1} \overline{\bar{V}}_{t+l-1}+\pi_{2} \overline{\bar{V}}_{t+l-2}+\cdots+\pi_{\mathrm{n}} \overline{\bar{V}}_{t+l-n}
$$

where $\pi$ weights are obtained by inverted form of the $\operatorname{ARMA}(2,1)$ model

$$
\left\{\begin{array}{l}
\pi_{1}=\phi_{1}-\theta \\
\pi_{j}=\phi_{2} \theta^{j-2}+\phi_{j} \theta^{j-1}-\theta^{j} \quad(j=1,2, \ldots, n)
\end{array}\right.
$$

Because the $\pi$ weights series is declined sharply, the accuracy of predicted values is sufficient in moderate $n$ previous values. The predicted wind speed with 24 lead time hours $\hat{V}_{\text {predited }}(24)$ is showed in Fig. 10, as example for March $7^{\text {th }}$, 2015. The wind speed forecast decays exponentially to mean value in long lead time. The error of wind speed forecast $\hat{V}_{\text {error }}$ (24) is randomly fluctuation in acceptable range, exception of too low and suddenly varied values. Almost actual wind speed is on $95 \%$ probability limits of $\hat{V}_{t}(l)$, which is variance of the wind speed forecast errors.

However, the uncorrelated wind speed forecast errors are decayed at longer lead times $l$. It is need to use $\psi$ weights series for updating the old predicted values $\hat{V}_{t}(l+k)$ at origin time $\mathrm{t}$ and lead time $l+\mathrm{k}$ by new ones $\widehat{V}_{t+k}(l)$ at origin time $\mathrm{t}+\mathrm{k}$ and lead time $l[2]$

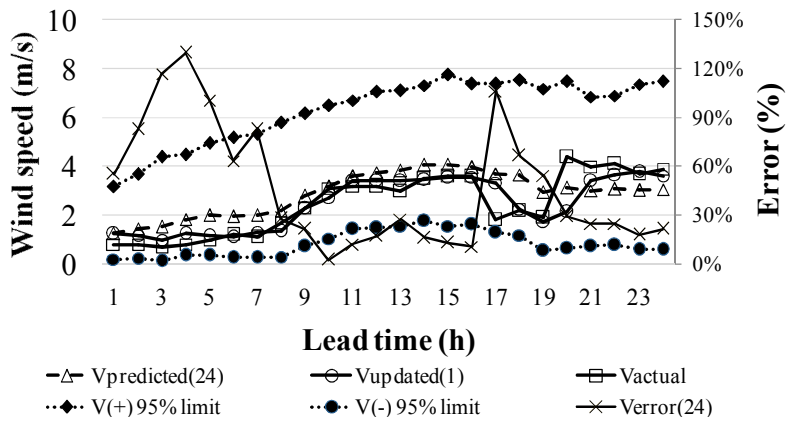

Fig. 10. The wind speed forecast in March $7^{\text {th }}, 2015$

$$
\widehat{V}_{t+k}(l)=\widehat{V}_{t}(l+k)+\psi_{l}\left\{\overline{\bar{V}}_{t+k}-\widehat{V}_{t}(\mathrm{k})\right\}
$$

where $\psi_{l}=\phi_{1} \psi_{l-1}+\phi_{2} \psi_{l-2}-\theta_{l} \quad(l=1,2, \ldots, n) \quad$ and $\psi_{0}=1, \psi_{l}=0$ for $l<0$ and $\theta_{l}=0$ for $l>1$.

In this case, the predicted wind speed is updated in every hour $\hat{V}_{\text {updated }}(1)$. The updated wind speed forecast sticks to the actual wind speed well (Fig. 10).

\section{Conclusion}

The HAWS data, which is transformed to stationary variable, is analyzed by Box - Jenkins methodology to build up wind speed models. Many subclass of ARMA(p,q) model is considered by autocorrelation, partial autocorrelation function and BIC criterion analysis to select the fitted models. Finally, in parameters estimating and diagnostic checking stage, $\operatorname{ARMA}(2,1)$ models are proposed for all months. The comparison between the observations and the simulations of autocorrelation function, frequency distribution and main statistical characteristics of demonstrates the models are fitted. These $\operatorname{ARMA}(2,1)$ models are used to generate reference monthly data which close to the actual statistical characteristics of the 3 year (from 2010 to 2012) time series of wind speed data in Jeju Island. These models are also developed to build up forecast model of wind speed in Jeju Island. In short-term, the wind speed forecast values are acceptable and keep on main characteristics of the models. It is useful tool for studying more in reliability analysis of power system including wind power in Jeju Island, Korea.

\section{Acknowledgements}

This work was supported by the Korean National Research Foundation (No. \#2012R1A2A2A01012803) and KOEN (Korea South-East Power Co., Ltd).

\section{References}

[1] B. Brown, R. Katz. and A. Murphy, "Times Series 
Models to Simulate and Forecast Wind Speed and Wind Power", Climate and applied meteorology, vol. 23, 1984

[2] Box P. and Jenkins M., Times Series Analysis, Forecasting and Control, Holden-Day, San Franc, 1976

[3] H. Nfaoui, and M. Sayigh, "Stochastic Simulation of Hourly Wind Speed Sequences in Tangiers", Solar Energy, vol. 56, no. 3, pp. 301-314, 1996

[4] K. Philippopoulos and D. Deligiorgi, "Statistical Simulation of Wind Speed in Athens, Greece Based on Weibull and ARMA Models" International Journal of Energy and Environment, issue.4, vol. 3, 2009

[5] P. Box, G. Cox, "An Analysis of Transformations", Jour. Royal Sta. Sco., B26, 211, 1964

[6] R. Karki, P. Hu and R. Billinton, "A simplified wind power generation model for reliability evaluation" IEEE Trans. Energy Conversion, vol. 21, no. 2, 2006.

[7] D. R. Chandra, M. S. Kumari, M. Sydulu, F. Grimaccia and M. Mussetta, "Adaptive wavelet neural network based wind speed forecasting studies" JEET, vol. 9, no. 6, pp. 1812-1821, 2014

[8] Dániel Divényi, János Divényi, "Wind Speed Simulator Base on Wind Generation Using Autoregressive Statistical Model", Electrotehnică, Electronică, Automatică, nr.2, 2012

[9] A. Kamjoo, A. Maheri, and G. Putrus, "Wind speed and solar irradiance variation simulation using ARMA models in design of hydrid wind-PV-battery system", Journal of clean energy technologies, vol. 1, no. 1,2013

[10] Lisa M. Bramer, Thesis: Method for modeling and forecasting wind characteristics, Iowa University, 2013

[11] M. Lei, L. Shiyuan, J. Chuanwen, L. Hongling, Z. Yan, "A Review on the Forecasting of Wind Speed And Generated Power", Renewable and Sustainable Energy Reviews, 2009

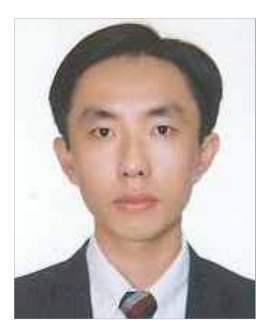

Duy-Phuong N.Do He was born in Can Tho city, VietNam in 1982. He received B.S and M.Sc degree in electrical engineering from CanTho University and HoChiMinh University of Technology, respectively. His research interest is stability evaluation of power system. Now, he is studying as $\mathrm{Ph} . \mathrm{D}$ student in Gyeongsang National University.

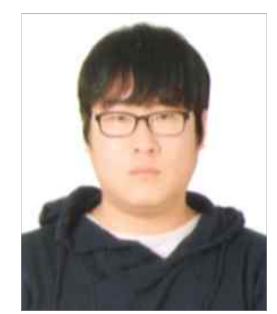

Yeonchan Lee He was born in Gosung, Korea in 1987. His research interest includes Transmission Expansion Planning using Reliability Evaluation of Power Systems. He received the B.Sc. degree from Gyeongsang National University in 2013.

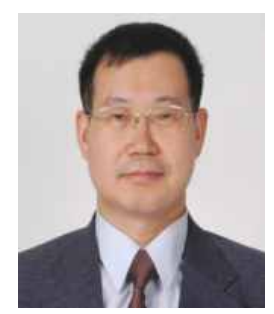

Jaeseok Choi $\mathrm{He}$ was born in Kyeongju, Korea, in 1958. He received the B.Sc., M.Sc., and Ph.D. degrees from Korea University, Seoul. Since 1991, he has been on the faculty of Gyeongsang National University, Jinju, Korea, where he is a professor. He was a visiting professor at Cornell University, Ithaca, NY, USA, in 2004. He is also adjunct professor at IIT, IL, USA since 2007. His research interests include fuzzy applications, probabilistic production cost simulation, reliability evaluation, and outage cost assessment of power systems. 